\title{
Dysbiosis of Gram-negative gut microbiota and the associated serum lipopolysaccharide exacerbates inflammation in type 2 diabetic patients with chronic kidney disease
}

\author{
MARIA V. SALGUERO ${ }^{1,2}$, MOHAMMED A. I. AL-OBAIDE ${ }^{1}$, RUCHI SINGH $^{1}$, \\ TIMO SIEPMANN $^{2,3}$ and TETYANA L. VASYLYEVA ${ }^{1}$
}

\begin{abstract}
${ }^{1}$ Department of Pediatrics, School of Medicine, Texas Tech University Health Sciences Center, Amarillo, TX 79106, USA; ${ }^{2}$ Center for Clinical Research and Management Education, Division of Health Care Sciences, Dresden International University, Dresden, Saxony D-01067; ${ }^{3}$ Department of Neurology, University Hospital Carl Gustav Carus, Technische Universitat Dresden, Dresden, Saxony D-01307, Germany
\end{abstract}

Received November 7, 2018; Accepted March 29, 2019

DOI: $10.3892 /$ etm.2019.7943

\begin{abstract}
Lipopolysaccharide (LPS), a potent endotoxin present in the outer membrane of Gram-negative bacteria, causes chronic immune responses associated with inflammation. In the present study, the association between LPS and the dysbiosis of Gram-negative bacteria in the gut microbiome was determined in patients with type 2 diabetes mellitus (T2DM) and chronic kidney disease (T2DM-CKD; stages 4 and 5, not on dialysis) compared with healthy individuals. Microbiome diversity was analyzed in patients with T2DM-CKD and healthy controls by sequencing the hypervariable sub-regions of the $16 \mathrm{~S}$ ribosomal RNA gene from stool samples. Serum samples were assayed by ELISA for LPS, C-reactive protein (CRP), tumor necrosis factor- $\alpha$ (TNF $\alpha$ ), interleukin-6 (IL6) and endothelin-1. A total of four gut Gram-negative phyla (Bacteroidetes, Proteobacteria, Fusobacteria and Verrucomicrobia) were identified in the gut microbiome of the T2DM-CKD and control groups. Proteobacteria, Verrucomicrobia and Fusobacteria exhibited significantly increased relative abundance in patients with T2DM-CKD when compared with controls $(\mathrm{P}<0.05)$. The levels of serum LPS were significantly increased in patients with T2DM-CKD compared with controls $(\mathrm{P}<0.05)$. Elevated serum LPS was significantly correlated with increased levels of TNF $\alpha$, IL6 and CRP. The dysbiosis of Gram-negative bacteria in the gut microbiome of patients with T2DM-CKD may contribute to the elevated serum levels of LPS and the consequential effects on the inflammatory biomarkers in
\end{abstract}

Correspondence to: Professor Tetyana L. Vasylyeva, Department of Pediatrics, School of Medicine, Texas Tech University Health Sciences Center, 1400 S Coulter Street, Amarillo, TX 79106, USA E-mail: tetyana.vasylyeva@ttuhsc.edu

Key words: chronic kidney disease, Gram-negative bacteria, gut microbiome, inflammatory markers, lipopolysaccharide, type 2 diabetes mellitus these patients. The association between the dysbiosis of Gram-negative bacteria in the gut microbiome of patients with T2DM-CKD, increased LPS levels and the effects on inflammatory biomarkers may provide insight into potential diagnostic and therapeutic approaches in the treatment of T2DM-CKD.

\section{Introduction}

Gut microbiota dysbiosis is associated with the development and progression of chronic kidney disease (CKD) $(1,2)$, cardiovascular complications (CVC) (2-4), obesity (5) and type 2 diabetes mellitus (T2DM) (5-8). High levels of serum lipopolysaccharide (LPS), a potent endotoxin produced by Gram-negative bacteria, have been associated with pathological processes, including diabetes, the progression of kidney disease, obesity and inflammation $(9,10)$. The release of LPS from Gram-negative bacteria in the gut and its passage to the blood causes LPS-associated toxicity (11). LPS induces inflammation via a cascade of inflammatory responses following the recognition of lipid A of LPS by immune cells $(12,13)$. Lipid A is the toxic component of LPS and serves as the microbe-specific molecular signal that binds to the surface receptor complexes of immune cells, which comprise Toll-like receptor 4 (TLR4) and myeloid differentiation factor 2 (MD2) $(14,15)$. The formation of the signaling complex can induce the activation of nuclear factor- $\kappa \mathrm{B}(\mathrm{NF}-\mathrm{\kappa} \mathrm{B})$. NF- $\mathrm{\kappa B}$ serves key role in signaling for the production of proinflammatory cytokines. Therefore, the detection of low quantities of circulating LPS by the TLR4 receptor system initiates the cascade of protein-protein interactions, leading to the production of proinflammatory cytokines $(16,17)$.

A number of factors associated with microbial alterations in the gut microbiota can lead to changes in host inflammatory responses (18). Diet and T2DM-associated medications are among the reported factors that alter the composition of the gut microbiome $(19,20)$. The consumption of foods high in fat increases the abundance of LPS-containing Gram-negative bacteria in the gut at the expense of Gram-positive bacteria $(20,21)$. Metformin, a blood glucose regulator, was 
reported to increase the relative abundance of LPS-producing Gram-negative gut bacteria (22). Numerous studies characterized the composition of gut microbiota in CKD $(23,24)$, and reported the correlation between LPS, increased intestinal permeability $(11,25)$ and inflammation in patients with CKD $(1,26)$. Metabolic syndrome and obesity are influenced by interactions between the gut microbiota and host genetics (27). Collectively, diet, the host genome and T2DM medication may be associated with imbalances in the gut microbiome. The multifactorial network between gut microbiome dysbiosis and the health of patients suggests an association between LPS, impaired gut permeability, cardiovascular diseases, CKD, and patients with T2DM and CKD (T2DM-CKD).

Of note, the aforementioned studies did not analyze patients with T2DM and advanced CKD (stages 4 and 5, not on dialysis). The present study investigated the link between gut Gram-negative bacteria and the derived LPS endotoxin, and the consequent inflammatory responses in patients with T2DM-CKD. We evaluated gut microbiome dysbiosis associated with LPS-producing bacteria in patients with T2DM-CKD compared with healthy control subjects. In addition, whether elevated serum LPS levels correlated with chronic inflammation was determined. Improved understanding in this research field is important for the development of effective therapeutic and diagnostic approaches for LPS-associated diseases.

\section{Patients and methods}

Study design. Healthy $(\mathrm{n}=20)$ and T2DM-CKD (stages 4 and 5, not on dialysis) $(n=20)$ subjects were $\geq 18$-years-old at enrollment into the present study. Healthy participants were referred by primary care physicians, and had no notable medical histories and were not on any medication. Patients with T2DM-CKD were recruited from the outpatient clinic of the Texas Tech University Health Sciences Center (Amarillo, TX, USA). Patients with T2DM-CKD with a glomerular filtration rate (GFR) of $<30 \mathrm{ml} / \mathrm{min} / 1.72 \mathrm{~m}^{2}$ and not on dialysis were enrolled in the present study. The exclusion criteria included patients treated with antibiotics for $\geq 3$ days during the month prior to the initiation of analysis, those diagnosed with end-stage liver disease or chronic gut-related diseases other than diabetes, pregnant, patients who previously had bariatric surgeries, and those with a mental status that restricted informed consent for enrollment. Criteria from Electronic Medical Records and Genomics (eMERGE) Network were used in categorizing patients for the study (28). The GFR was calculated using the CKD-EPI formula (29) and staging corresponded to the Kidney Disease Outcomes Quality Initiative guidelines (30). The Institutional Review Board of Texas Tech University Health Sciences Center approved the present study. Patients provided informed consent prior to study participation. The study design included analyses of the dysbiosis of Gram-negative bacteria in the gut microbiome, serum inflammatory markers, and endothelial dysfunction biomarkers in healthy patients and those with T2DM-CKD (stages 4 and 5, not on dialysis).

Fecal sample collection and DNA analysis. Stool samples were collected from subjects within $24 \mathrm{~h}$ prior to a clinical visit. The samples were processed for DNA extraction within 24 h using PSP Spin Stool DNA PLUS Kits (Invitek
Biotechnology and Biodesign LTD, Berlin, Germany) according to the manufacturer's protocols. DNA sequencing and gut microbiota classification were conducted as previously described (31-33). Briefly, the hypervariable sub-regions of $16 \mathrm{~S}$ ribosomal RNA genes were amplified by polymerase chain reaction with the bacterial universal primer sets, $341 \mathrm{~F}$ and $805 \mathrm{R}$, which contained Illumina adaptors. The obtained libraries were sequenced by MiSeq sequencer (Illumina Inc., San Diego, CA, USA) using a 600 cycle v3 sequencing kit. The Human 16S rRNA database was used to remove matching DNA sequences in the human genome. The microbiome operational taxonomic units (OTUs) were classified and compiled into the taxonomic levels as 'counts' and 'percentage' files.

Biomarkers assays. ELISA kits were employed to assay biomarkers in the serum from the blood of fasting patients and healthy controls. ELISA kits (R\&D Systems, Minneapolis, MN, USA) were used to assay endothelin-1 (ET-1), C-reactive protein (CRP), tumor necrosis factor- $\alpha$ (TNF $\alpha)$, and interleukin-6 (IL6). An ELISA kit obtained from TSZ ELISA (Waltham, MA, USA) was used to LPS levels.

Statistical analysis. Excel Data Analysis Toolpack and GraphPad Prism 7.01 (GraphPad Software, Inc., La Jolla, CA, USA) were used for the statistical analysis of various parameters via paired or unpaired t-tests, and two-way analysis of variance (ANOVA) with Bonferroni's post hoc test. A Mann-Whitney test was used to compare data between patients with T2DM-CKD and healthy controls. Regression and Spearman correlation analyses were used to assess the strength and direction of correlations between LPS and serum biomarkers. $\mathrm{P}<0.05$ was considered to indicate a statistically significant difference. To demonstrate the significance of the source of variations in the abundance of identified OTUs between the two groups, two-way ANOVA with an adjusted P-value (multiplicity test) was applied for each comparison. Multiplicity tests revealed three adjusted P-values, interaction $[\mathrm{p}(\mathrm{I})]$, column factor $[\mathrm{p}(\mathrm{CF})]$ and row factor $[\mathrm{p}(\mathrm{RF})]$.

\section{Results}

Clinical characteristics. The healthy controls and patients with T2DM-CKD exhibited no significant differences in gender, age and body mass index values (Table I). Significant differences were observed between the control and T2DM-CKD groups for total cholesterol, triglycerides, and low- and high-density lipoprotein. Insulin dependence among the diabetic group was $40 \%$ at enrollment. The remaining patients of the group had received sitagliptin or glipizide. None of the patients with T2MD-CKD were administered metformin. Patients in the T2DM-CKD group used ranitidine (10\% of the group) and proton pump inhibitors (20\% of the group). Antihypertensive medications were prescribed to $90 \%$ of the recruited patients with T2MD-CKD (mono or combined therapy), with $40 \%$ on angiotensin-converting enzyme inhibitors and $20 \%$ on angiotensin II receptor blockers. A total of $40 \%$ of the T2MD-CKD group were taking statins.

Diversity of LPS producing gut Gram-negative bacteria in the T2DM-CKD and control groups. We identified four 
Table I. Clinical parameters of patients with T2DM-CKD and control participants recruited in the present study.

\begin{tabular}{lccc}
\hline Parameter & T2DM-CKD & Control & P-value \\
\hline Sex & 9 male, 11 female & 11 male, 9 female & 0.75 \\
Age (years) & $62.8 \pm 3.6$ & $58.5 \pm 4.1$ & 0.23 \\
BMI $\left(\mathrm{kg} / \mathrm{m}^{2}\right)$ & $32.7 \pm 3.8$ & $29.4 \pm 2.6$ & 0.18 \\
eGFR $\left(\mathrm{ml} / \mathrm{min} / 1.72 \mathrm{~m}^{2}\right)$ & $16.54 \pm 3.01$ & $>60$ & $\mathrm{~N} / \mathrm{A}$ \\
Proteinuria $(\mathrm{g} / 24 \mathrm{~h})$ & $3.58 \pm 2.29$ & $\mathrm{~N} / \mathrm{A}$ & $\mathrm{N} / \mathrm{A}$ \\
HDL $(\mathrm{mg} / \mathrm{dl})$ & $38.9 \pm 6.2$ & $58.5 \pm 8.4$ & 0.01 \\
LDL $(\mathrm{mg} / \mathrm{dl})$ & $112 \pm 12.7$ & $81 \pm 9.6$ & 0.01 \\
Total cholesterol $(\mathrm{mg} / \mathrm{dl})$ & $161.7 \pm 15.2$ & $189.0 \pm 10.7$ & 0.01 \\
Triglycerides $(\mathrm{mg} / \mathrm{dl})$ & $193.1 \pm 22.7$ & $82.0 \pm 17.1$ & 0.01 \\
\hline
\end{tabular}

T2DM-CKD, type 2 diabetes mellitus and chronic kidney disease; BMI, body mass index; LDL, low-density lipoprotein; HDL, high-density lipoprotein; N/A, not calculated. The data presented as mean \pm standard deviation.
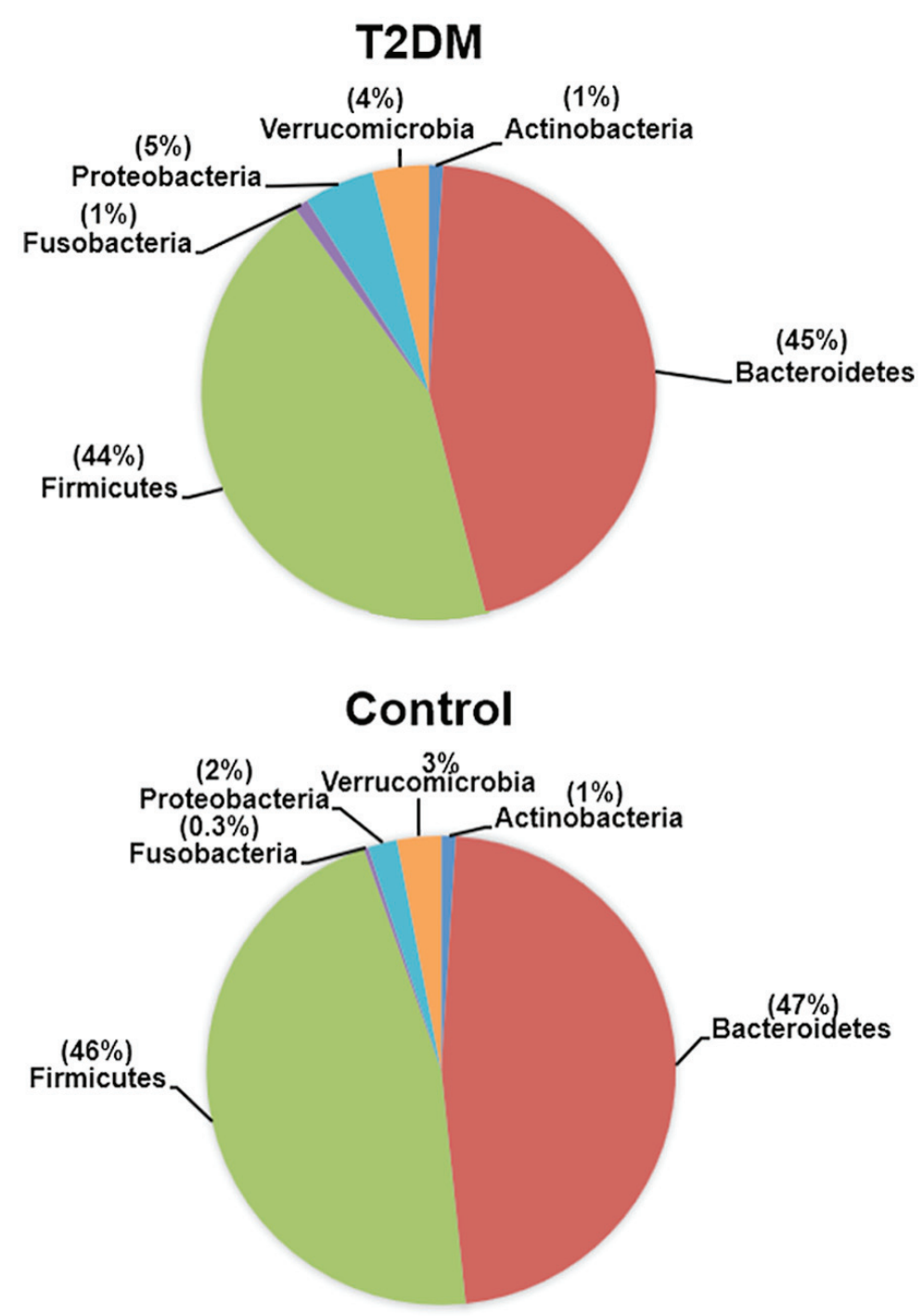

Figure 1. Identified gut microbiota phyla in patients with T2DM-CKD and healthy individuals. The relative abundances were expressed as mean percentages. T2DM, type 2 diabetes mellitus.

Gram-negative phyla in the gut microbiota of the T2DM-CKD and control groups, including Bacteroidetes, Proteobacteria, Verrucomicrobia and Fusobacteria. Of the Gram-positive phyla, Firmicutes and Actinobacteria were identified; the phyla exhibited a mean percentage of $\geq 0.3 \%$ (Fig. 1). The relative abundance of each phylum in the two study 

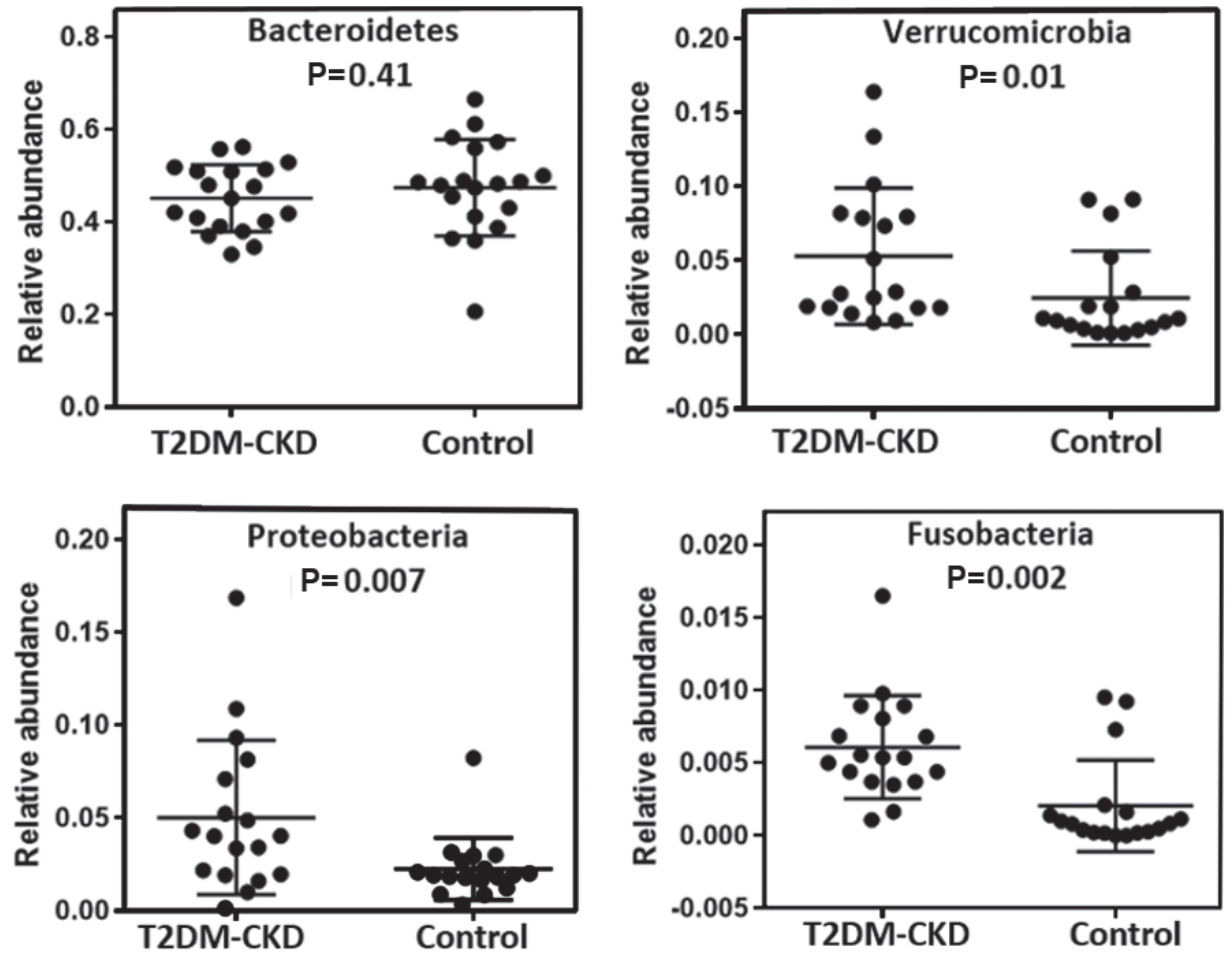

Figure 2. Relative abundance of four gut Gram-negative phyla in the T2DM-CKD and control groups. No significant difference was observed between the relative abundance of Bacteroidetes. Significant differences were observed in the relative abundance of the three Gram-negative phyla Proteobacteria, Verrucomicrobia and Fusobacteria between the two groups. P<0.05. T2DM-CKD, type 2 diabetes mellitus and chronic kidney disease.

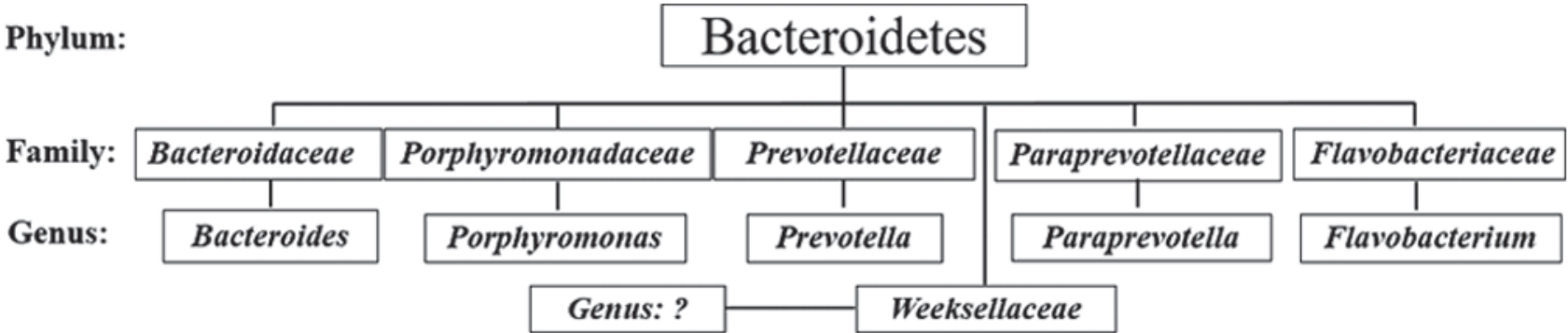

Figure 3. Identified families and genera in the Gram-negative phylum Bacteroidetes in the type 2 diabetes mellitus-chronic kidney disease and control groups with high relative abundances.

groups, T2DM-CKD and control, revealed no significant difference for possessing Bacteroidetes (Fig. 2). Within the Bacteroidetes, five families were identified, including Bacteroidaceae, Porphyromonadaceae, Prevotellaceae, Paraprevotellaceae and Flavobacteriaceae; five genera (Bacteroides, Porphyromonas, Prevotella, Paraprevotella and Flavobacterium) were also reported in the human gut (Fig. 3). In addition, bacteria of a sixth family, Weeksellaceae in the phylum Bacteroidetes, were detected (Fig. 3). To the best our knowledge, Weeksellaceae has not been reported in the human gut microbiota, but has been identified in human saliva. Furthermore, the results of the present study revealed that members of the Bacteroides genus of the Bacteroidetes phylum were dominant in the gut microbiota of patients with T2DM-CKD (36\%) and controls (34\%); however, no significant difference in the mean percentages was observed.

The three Gram-negative phyla (Proteobacteria, Verrucomicrobia and Fusobacteria) exhibited lower mean percentages (0.3-5\%) than Bacteroidetes in the T2DM-CKD and control groups (Fig. 1). As presented in Fig. 2, Mann-Whitney analysis demonstrated significantly increased relative abundance of the three aforementioned Gram-negative phyla in the gut of patients with T2DM-CKD compared with the control. A total of 17 genera in the Gram-negative phylum Proteobacteria and 10 genera within the family Enterobacteriaceae were reported (Fig. 4A). Akkermansia was identified in the Gram-negative phylum Verrucomicrobia (Fig. 4B) and two genera, Leptotrichia and Fusobacterium, were detected in the Gram-negative phylum Fusobacteria (Fig. 4C).

Multiplicity tests with adjusted P-values revealed the abundance of the identified OTUs between the two study groups, T2DM-CKD and controls. Analysis demonstrated a significant difference in the specified source of variation for the relative abundance of Proteobacteria and Fusobacteria between the T2DM-CKD and control groups $[\mathrm{p}(\mathrm{I})=0.0226, \mathrm{p}(\mathrm{RF})<0.0001$ 
A

Phylum: $\quad$ Proteobacteria
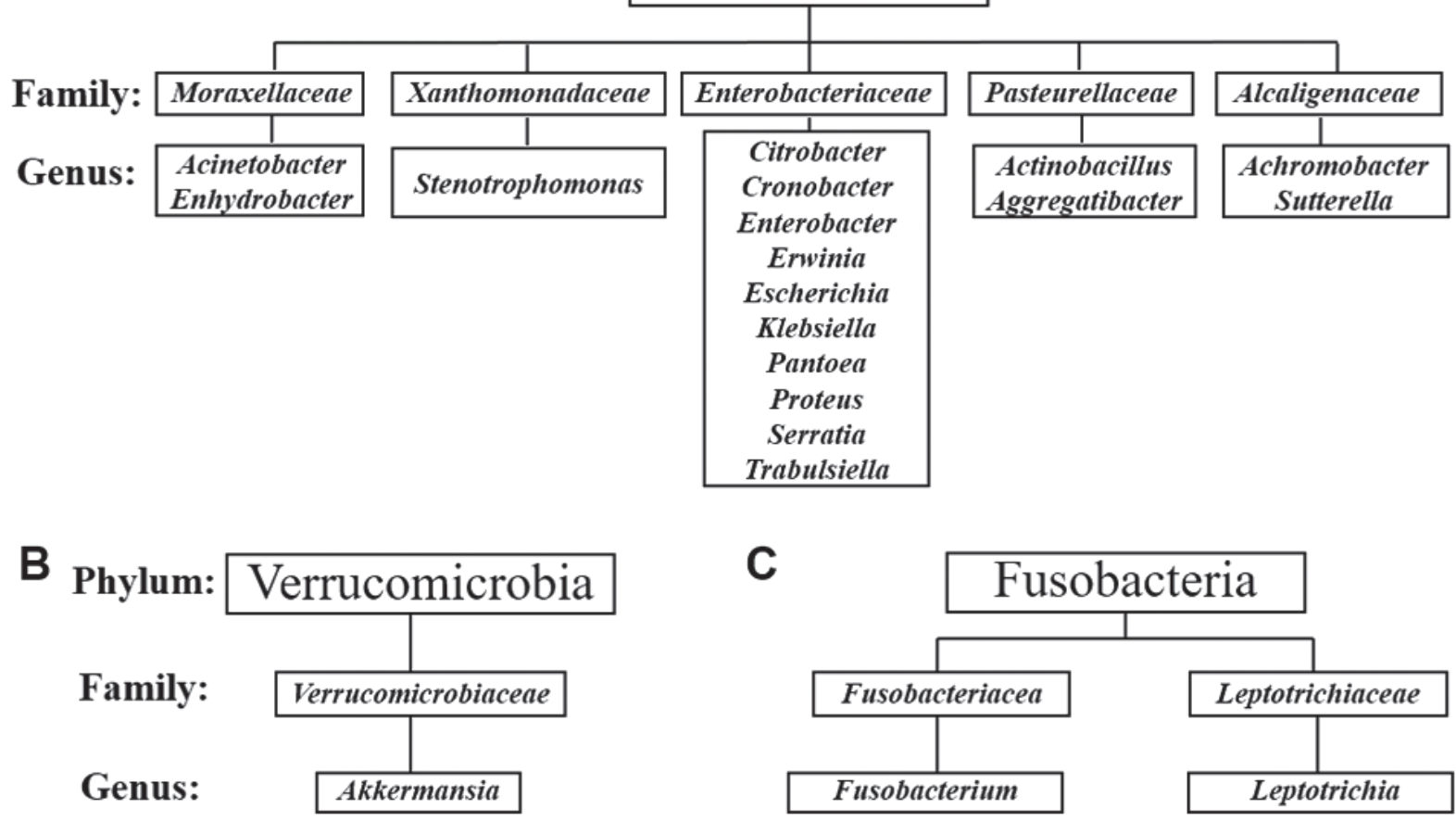

Figure 4. Members of the gut microbiota identified in three Gram-negative phyla with low relative abundances within patients with type 2 diabetes mellitus and controls. (A) The phylum Proteobacteria comprised of 5 families and 17 genera. (B) A single genus was identified in the phylum, Verrucomicrobia; (C) 2 genera were identified in the phylum, Fusobacteria.

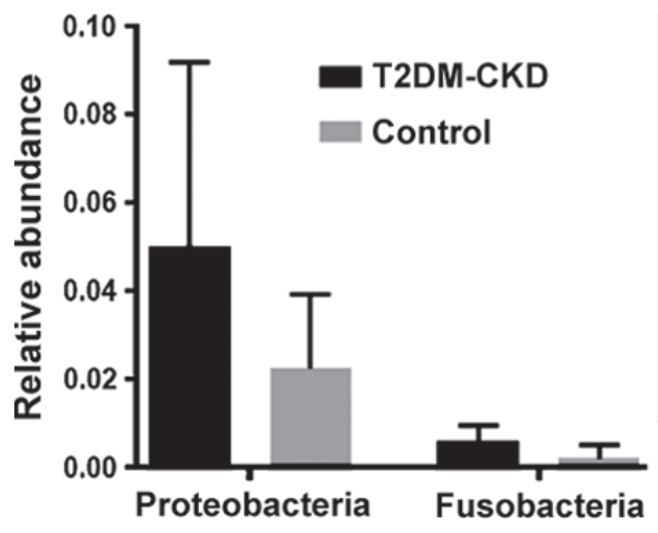

\begin{tabular}{|c|c|c|c|c|}
\hline $\begin{array}{c}\text { Source of } \\
\text { variation }\end{array}$ & $\begin{array}{c}\% \text { of } \\
\text { total } \\
\text { variation }\end{array}$ & P-value & $\begin{array}{c}\text { P-value } \\
\text { summary }\end{array}$ & Significant? \\
\hline Interaction & 4.142 & 0.0297 & $\star$ & Yes \\
\hline $\begin{array}{c}\text { Row } \\
\text { factor }\end{array}$ & 31.24 & $<0.0001$ & $\star \star \star \star$ & Yes \\
\hline $\begin{array}{c}\text { Column } \\
\text { factor }\end{array}$ & 7.475 & 0.004 & $\star \star$ & Yes \\
\hline
\end{tabular}

Figure 5. Multiple comparison analysis with adjusted P-values of the relative abundances of Proteobacteria and Fusobacteria in patients with T2DM and controls. The two phyla demonstrated a significant difference for the specified source of variations. P $<0.05$. T2DM-CKD, type 2 diabetes mellitus and chronic kidney disease.

and $\mathrm{p}(\mathrm{CF})=0.0184$; Fig. 5]. On the contrary, the multiplicity tests indicated no significant differences in the abundance of Proteobacteria and Verrucomicrobia, or Verrucomicrobia and Fusobacteria between the T2DM-CKD and control groups.

Correlation between elevated levels of serum LPS and the assayed biomarkers in the T2DM-CKD group. The significant difference observed in the relative abundance of gut Gram-negative bacteria in patients with T2DM-CKD compared with the control group may be associated with increased levels of serum LPS. ELISA demonstrated that serum LPS levels were significantly higher in patients with T2DM-CKD compared with the controls ( $\mathrm{P} \leq 0.05$; Fig. 6A).
Then, the correlation between elevated serum LPS levels and inflammatory biomarkers was determined by linear regression and Spearman correlation analyses. Linear regression was performed using circulating inflammatory biomarkers as a dependent variable and LPS as an independent variable. The results demonstrated that elevated LPS levels were associated with increased levels of CRP, IL6 and TNF $\alpha$ in the blood of patients with T2DM-CKD (P $\leq 0.05$; Fig. 6B-D). Whereas, LPS and endothelial dysfunction biomarker, ET-1, exhibited a non-significant correlation. The levels of inflammatory biomarkers were significantly decreased in healthy individuals compared with patients with T2DM-CKD and exhibited a non-significant correlation with LPS. 

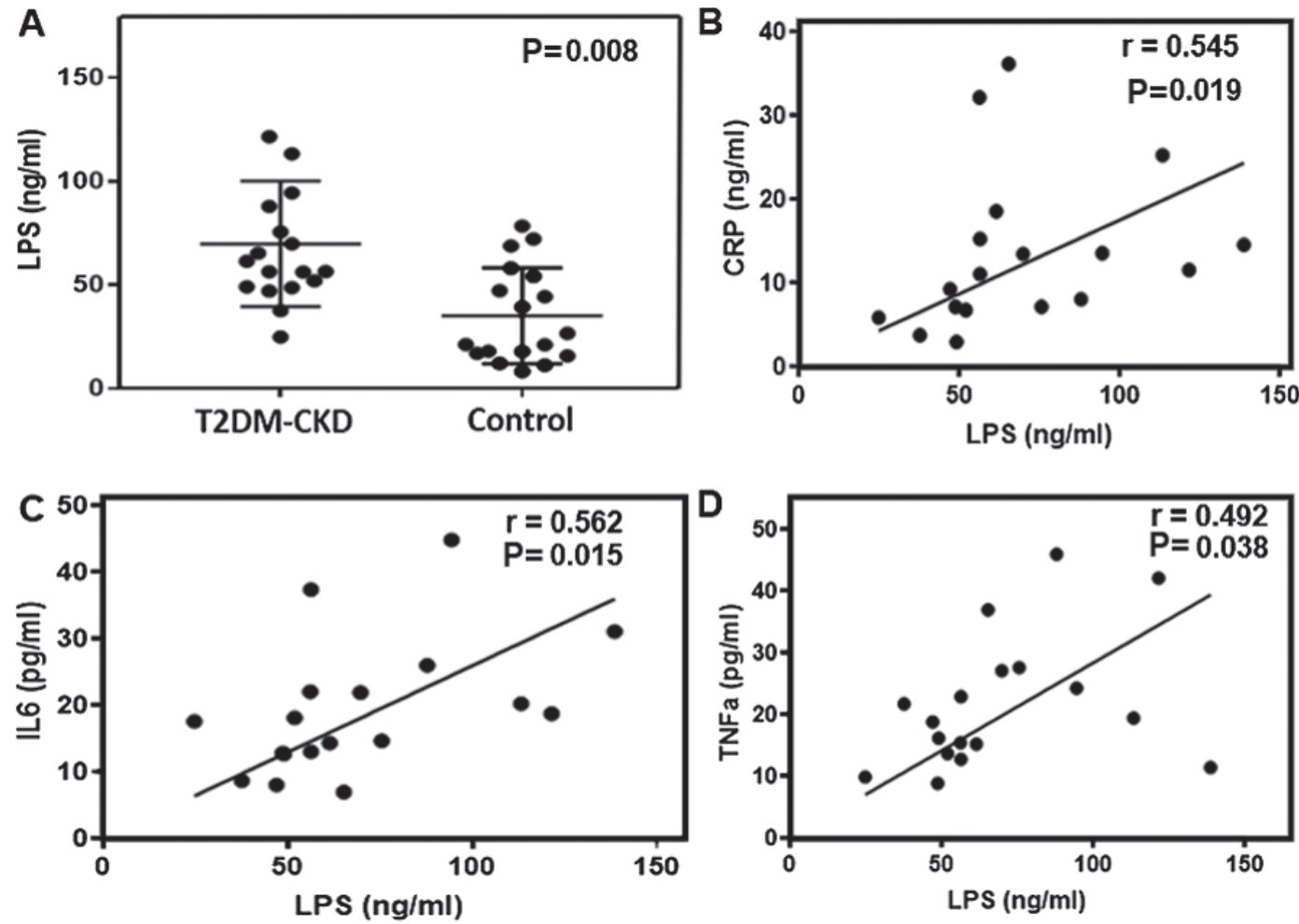

Figure 6. Correlation between elevated serum LPS and inflammatory biomarkers in the T2DM-CKD and control groups. (A) Serum LPS (ng/ml) levels in T2DM-CKD patients compared with the controls. (B-D) Elevated serum levels of LPS revealed a significant positive correlation with (B) CRP, (C) IL6 and (D) TNF $\alpha$ in the blood of patients with T2DM-CKD. P<0.05. CRP, C-reactive protein; IL6, interleukin-6; LPS, lipopolysaccharide; T2DM-CKD, type 2 diabetes mellitus and chronic kidney disease; TNF $\alpha$, tumor necrosis factor $\alpha$.

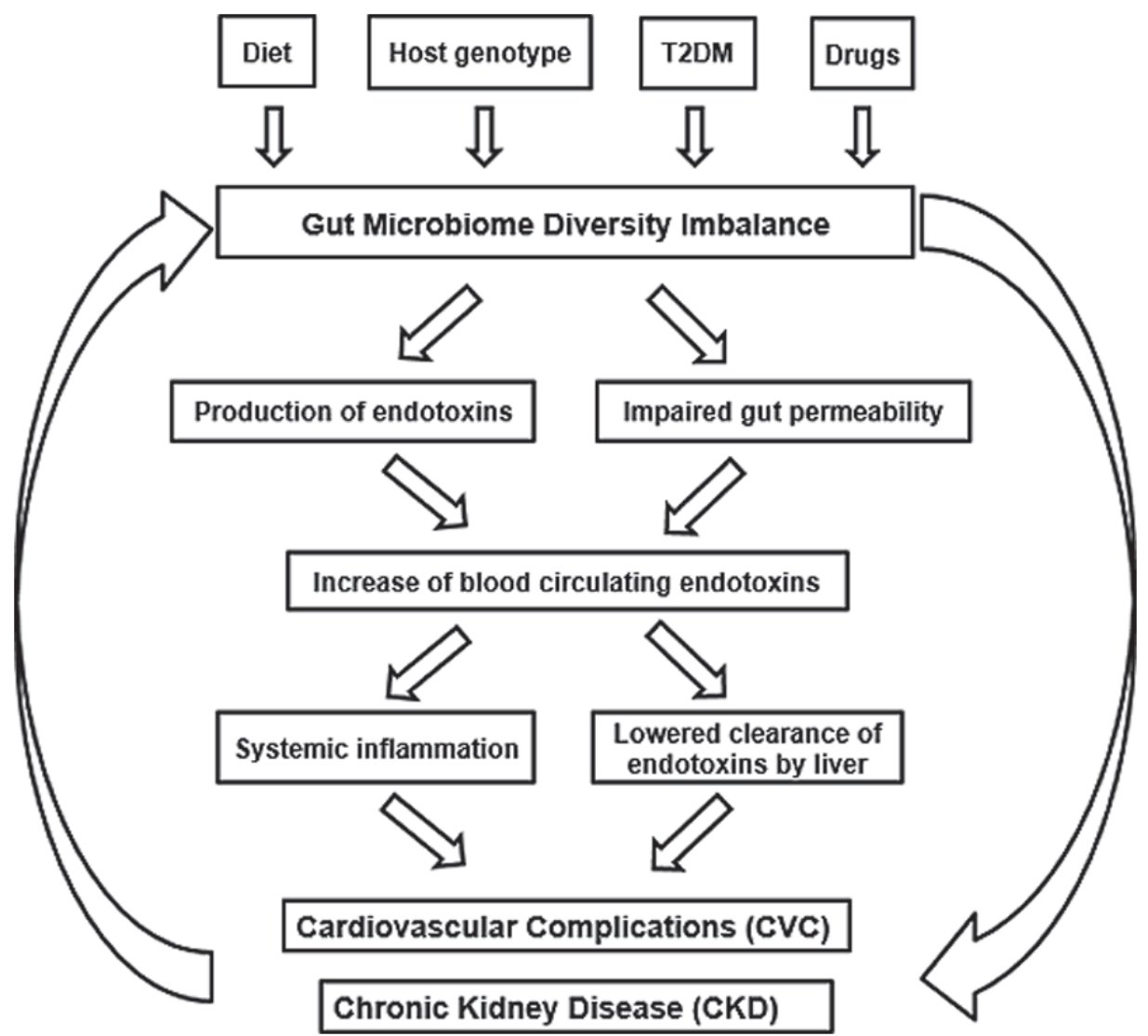

Figure 7. Multifactorial effects of host genotype, diet, drugs and the potential influence of T2DM on alterations in the gut microbiome, which is followed by a cascade of events that promote systemic inflammation and reduces the clearance of endotoxins associated with chronic kidney disease. T2DM, T2DM type 2 diabetes mellitus. 


\section{Discussion}

The results demonstrated a shift in the gut microbial community in patients with T2DM-CKD towards increased relative abundances of Gram-negative bacteria. The dysbiosis of Gram-negative gut microbiota is a health risk associated with elevated LPS levels and correlated with increased levels of inflammatory biomarkers that can cause chronic inflammation in patients with T2DM-CKD. LPS forms the outer monolayer of the outer membranes of the majority of Gram-negative bacteria with profound effects on the immune system $(10,34,35)$. Nicholson et al (18) reported the involvement of bacterial LPS in metabolic and numerous inflammatory disorders (18).

A total of fourGram-negative phyla,including Bacteroidetes, Proteobacteria, Verrucomicrobia and Fusobacteria were identified in the gut microbiota of T2DM-CKD and control subjects. Previous studies proposed Bacteroidetes as the primary Gram-negative microbiota in the gastrointestinal tract $(36,37)$. The present study identified five previously reported genera in the phylum Bacteroidetes, including Porphyromonas, Prevotella, Paraprevotella and Flavobacterium $(38,39)$. Members of the Weeksellaceae family in the Bacteroidetes phylum had been detected in human saliva but were reported not to inhabit the human gut (40). In the present study, members of the genus Bacteroides were reported to be dominant in the gut microbiota of the T2DM-CKD (stages 4 and 5, not on dialysis) and control groups; no significant differences were observed. Jiang et al (41) revealed that members of the genus Bacteroides were prevalent in samples collected from patients with end-stage renal disease.

The other three Gram-negative phyla, Proteobacteria, Verrucomicrobia and Fusobacteria exhibited reduced abundances in the T2DM-CKD group compared with that of Bacteroidetes. The mean percentages for Proteobacteria (5\%), Verrucomicrobia (4\%) and Fusobacteria $(1 \%)$ in patients with T2DM-CKD were significantly higher than the relative abundance in the controls (2, 3 and $0.3 \%$, respectively). A total of 17 genera in five families in the phylum Proteobacteria were identified. Several of the identified genera were within the family Enterobacteriaceae. Of note, the activity of LPS in Bacteroidetes is less effective compared with that of other Gram-negative bacteria. Numerous studies reported that Escherichia coli and other bacteria in the Enterobacteriaceae family possess markedly increased LPS endotoxin activity than LPS extracted from the envelope of Bacteroides fragilis of the Bacteroidetes phylum $(42,43)$. LPS is structurally similar to lipid A of E. coli; however, lipid A of B. fragilis differs by the lack of a phosphate group on $\mathrm{C} 4$ of the non-reducing amino sugar, and by the presence of fewer and various fatty acids (42). These differences may account for the low endotoxic activity of $B$. fragilis-derived LPS. In addition, Bacteroides fragilis endotoxins are a notable cause of anaerobic bacteremia and sepsis (44). The present study also reported the three genera, Akkermansia, Fusobacterium and Leptotrichia which belong to two phyla, Verrucomicrobia and Fusobacteria, are a part of the human gut microbiota. Recent studies reported the genera identified in the present study, including, Leptotrichia (45), Akkermansia $(46)$ and Fusobacterium $(45,47)$ to be a part of the human gut flora.
Additionally, the present study revealed a significant correlation between elevated levels of LPS, and inflammatory biomarkers, including CRP, TNF $\alpha$ and IL-6 in patients with T2DM-CKD compared with the controls. Increased levels of circulating LPS have been reported to promote systemic inflammation $(42,43)$. The inflammatory response is initiated following the recognition of lipid A by TLR4 and MD2 of immune cells (12-15). The formation of the TLR4-MD-2 complex activates the signaling pathway controlling the expression of various inflammatory genes (48-51). Thus, when the levels of LPS in the blood exceed a threshold concentration, myeloid cells are systemically activated to produce excessive quantities of pro-inflammatory cytokines, including IL-1 $\beta$, IL-6 and TNF $\alpha(52,53)$.

An additional factor contributing to elevated LPS in the blood is diet. Consuming foods high in fat promotes Gram-negative bacteria in the gut $(20,21)$. In the present study, it was observed that patients with T2DM-CKD consumed more fat than control participants. Furthermore, several studies revealed the effects of the gut microbiota in regulating the integrity of the intestine permeability; gut permeability is critical for health $(54,55)$. Gut bacteria can induce alterations in zonulin, an essential component of the intercellular tight junction and an important factor associated with gut permeability $(56,57)$. The loss of integrity of intestinal permeability allows the translocation of LPS from the gut into the blood (58-61). Thus, the loss of gut wall integrity associated with gut microbiota dysbiosis could increase the levels of circulating LPS in patients with T2DM-CKD.

Numerous cross-sectional studies reported that insulin resistance and T2DM are associated with increased levels of CRP, IL-6 and TNF $\alpha$, markers of subclinical systemic inflammation (62). The precise mechanisms underlying the upregulation of these inflammatory markers require further investigation; however, the inflammatory response under the conditions of T2DM may be multifactorial. The present study aimed to demonstrate LPS as a major factor in the onset of inflammation; however, other factors, including age, quantity of adipose tissue and advanced glycation end products may serve a role. Furthermore, the host genome and drugs have been associated with gut microbiome imbalances $(19,20,27)$. The present study proposed a multifactorial association between the composition of the gut microbiome and the health of patients with T2DM-CKD (Fig. 7), including the contribution of T2DM, LPS endotoxin and impaired gut permeability in the development of CVC, CKD and T2DM-CKD.

However, there are two limitations to this study. First, using a small number of subjects is not to quantify the general performance of dysbiosis within T2DM -CKD patients but to document the existence of an effect (63). The second limitation is the analysis of the potential association of only four Gram-negative phyla with LPS. These phyla predominantly exhibited a mean percentage of greater than or equal to $0.3 \%$.

In conclusion, the present study revealed the dysbiosis of three Gram-negative phyla, including Proteobacteria, Verrucomicrobia and Fusobacteria in the gut microbiota of patients with T2DM-CKD, which was associated with elevated levels of LPS endotoxin in the blood. Direct therapeutic interventions are required to reduce the relative abundance of LPS-producing bacteria in patients with T2DM-CKD. 


\section{Acknowledgements}

This work is a part of MVS master's thesis of the Master's Program in Clinical Research, Center for Clinical Research and Management Education, Division of Health Care Sciences, Dresden International University, Dresden, Germany. The authors would like to acknowledge the assistance of Mrs Ibtisam Al-Obaidi (School of Medicine, Texas Tech University Health Sciences Center, Amarillo, Texas, USA) for technical services, Dr Kameswara Rao Kottapalli (Center for Biotechnology and Genomics, Texas Tech University, Lubbock, Texas, USA) for DNA sequencing, and Dr Candace A. Myers and Ms Noel Howard (School of Medicine, Texas Tech University Health Sciences Center, Amarillo, Texas, USA) for editing the manuscript.

\section{Funding}

No funding was received.

\section{Availability of data and materials}

All data generated or analyzed during this study are included in this published article.

\section{Authors' contributions}

TS and TLV conceived, designed and supervised the study. MVS, MAIAO and RS performed the experiments and analyzed the data. MVS, MAIAO, TS and TLV wrote and revised the manuscript. All authors read and approved the final manuscript.

\section{Ethics approval and consent to participate}

The Institutional Review Board of Texas Tech University Health Sciences Center/Amarillo, Texas approved the protocol, which was performed in accordance with the Declaration of Helsinki. Written informed consent was obtained from each participant prior to participation in the study.

\section{Patient consent for publication}

Not applicable.

\section{Competing interests}

The authors declare that they have no competing interests.

\section{References}

1. Chaves LD, McSkimming DI, Bryniarski MA, Honan AM, Abyad S, Thomas SA, Wells S, Buck M, Sun Y, Genco RJ, et al: Chronic kidney disease, uremic milieu, and its effects on gut bacterial microbiota dysbiosis. Am J Physiol Renal Physiol 315: F487-F502, 2018.

2. Mafra D, Lobo JC, Barros AF, Koppe L, Vaziri ND and Fouque D: Role of altered intestinal microbiota in systemic inflammation and cardiovascular disease in chronic kidney disease. Future Microbiol 9: 399-410, 2014.

3. Bu J and Wang Z: Cross-Talk between gut microbiota and heart via the routes of metabolite and immunity. Gastroenterol Res Pract 2018: 6458094, 2018
4. Tang WW and Hazen SL: The contributory role of gut microbiota in cardiovascular disease. J Clin Invest 124: 4204-4211, 2014.

5. Harsch IA and Konturek PC: The role of gut microbiota in obesity and type 2 and type 1 diabetes mellitus: New insights into 'old' diseases. Med Sci (Basel) 6: pii: E32, 2018.

6. Gomes JMG, Costa JA and Alfenas RCG: Metabolic endotoxemia and diabetes mellitus: A systematic review. Metabolism 68: 133-144, 2017.

7. Sabatino A, Regolisti G, Cosola C, Gesualdo L and Fiaccadori E: Intestinal microbiota in type 2 diabetes and chronic kidney disease. Curr Diab Rep 17: 16, 2017.

8. Fallucca F, Porrata C, Fallucca S and Pianesi M: Influence of diet on gut microbiota, inflammation and type 2 diabetes mellitus. First experience with macrobiotic Ma-Pi 2 diet. Diabetes Metab Res Rev 30 (Suppl 1): S48-S54, 2014.

9. Harley IT and Karp CL: Obesity and the gut microbiome: Striving for causality. Mol Metab 1: 21-31, 2012.

10. Raetz CR and Whitfield C: Lipopolysaccharide endotoxins. Annu Rev Biochem 71: 635-700, 2002.

11. Guo S, Al-Sadi R, Said HM and Ma TY: Lipopolysaccharide causes an increase in intestinal tight junction permeability in vitro and in vitro and in vivo by inducing enterocyte membrane expression and localization of TLR-4 and CD14. Am J Pathol 182: 375-387, 2013.

12. Tidswell M, Tillis W, Larosa SP, Lynn M, Wittek AE, Kao R, Wheeler J, Gogate J and Opal SM; Eritoran Sepsis Study Group: Phase 2 trial of eritoran tetrasodium (E5564), a toll-like receptor 4 antagonist, in patients with severe sepsis. Crit Care Med 38: 72-83, 2010.

13. Bohannon JK, Hernandez A, Enkhbaatar P, Adams WL and Sherwood ER: The immunobiology of toll-like receptor 4 agonists: From endotoxin tolerance to immunoadjuvants. Shock 40: 451-462, 2013.

14. Dauphinee SM and Karsan A: Lipopolysaccharide signaling in endothelial cells. Lab Invest 86: 9-22, 2006.

15. Carpenter S and O'Neill LA: Recent insights into the structure of Toll-like receptors and post-translational modifications of their associated signalling. Biochem J 422: 1-10, 2009.

16. Kuzmich NN, Sivak KV, Chubarev VN, Porozov YB, Savateeva-Lyubimova TN and Peri F: TLR4 signaling pathway modulators as potential therapeutics in inflammation and sepsis. Vaccines (Basel) 5: pii: E34, 2017.

17. Wang F, Liu J, Weng T, Shen K, Chen Z, Yu Y, Huang Q, Wang G, Liu $Z$ and Jin S: The inflammation induced by lipopolysaccharide can be mitigated by short-chain fatty acid, butyrate, through upregulation of IL-10 in septic shock. Scand J Immunol 85: 258-263, 2017.

18. Nicholson JK, Holmes E, Kinross J, Burcelin R, Gibson G, Jia W and Pettersson S: Host-gut microbiota metabolic interactions. Science 336: 1262-1267, 2012.

19. Wu H, Esteve E, Tremaroli V, Khan MT, Caesar R, Mannerås-Holm L, Ståhlman M, Olsson LM, Serino M, Planas-Fèlix M, et al: Metformin alters the gut microbiome of individuals with treatment-naive type 2 diabetes, contributing to the therapeutic effects of the drug. Nat Med 23: 850-858, 2017.

20. Ahola AJ, Lassenius MI, Forsblom C, Harjutsalo V, Lehto M and Groop PH: Dietary patterns reflecting healthy food choices are associated with lower serum LPS activity. Sci Rep 7: 6511, 2017.

21. Cani PD, Neyrinck AM, Fava F, Knauf C, Burcelin RG Tuohy KM, Gibson GR and Delzenne NM: Selective increases of bifidobacteria in gut microflora improve high-fat-diet-induced diabetes in mice through a mechanism associated with endotoxaemia. Diabetologia 50: 2374-2383, 2007.

22. Montandon SA and Jornayvaz FR: Effects of antidiabetic drugs on gut microbiota composition. Genes (Basel) 8: pii: E250, 2017.

23. Al Khodor S and Shatat IF: Gut microbiome and kidney disease: A bidirectional relationship. Pediatr Nephrol 32: 921-931, 2017.

24. Vaziri ND, Wong J, Pahl M, Piceno YM, Yuan J, DeSantis TZ, Ni Z, Nguyen TH and Andersen GL: Chronic kidney disease alters intestinal microbial flora. Kidney Int 83: 308-315, 2013.

25. Forsyth CB, Shannon KM, Kordower JH, Voigt RM, Shaikh M, Jaglin JA, Estes JD, Dodiya HB and Keshavarzian A: Increased intestinal permeability correlates with sigmoid mucosa alpha-synuclein staining and endotoxin exposure markers in early Parkinson's disease. PLoS One 6: e28032, 2011.

26. Terawaki H, Yokoyama K, Yamada Y, Maruyama Y, Iida R, Hanaoka K, Yamamoto H, Obata T and Hosoya T: Low-grade endotoxemia contributes to chronic inflammation in hemodialysis patients: Examination with a novel lipopolysaccharide detection method. Ther Apher Dial 14: 477-482, 2010. 
27. Ussar S, Griffin NW, Bezy O, Fujisaka S, Vienberg S, Softic S, Deng L, Bry L, Gordon JI and Kahn CR: Interactions between gut microbiota, host genetics and diet modulate the predisposition to obesity and metabolic syndrome. Cell Metab 22: 516-530, 2015.

28. Richesson RL, Rusincovitch SA, Wixted D, Batch BC, Feinglos MN, Miranda, ML, Hammond WE, Califf RM and Spratt SE: A comparison of phenotype definitions for diabetes mellitus. J Am Med Inform Assoc 20: e319-e326, 2013.

29. National Kidney Foundation: GFR calculator. www.kidney.org/ professionals/kdoqi/gfr_calculator. Accessed, August 9, 2017.

30. National Kidney Foundation: K/DOQI clinical practice guidelines for chronic kidney disease: Evaluation, classification, and stratification. Am J Kidney Dis 39 (Suppl 1): S1-S266, 2002.

31. Al-Obaide MAI, Singh R, Datta P, Rewers-Felkins KA, Salguero MV, Al-Obaidi I, Kottapalli KR and Vasylyeva TL: Gut microbiota-dependent trimethylamine-N-oxide and serum biomarkers in patients with T2DM and advanced CKD. J Clin Med 6: pii: E86, 2017.

32. Hiergeist A and Reischl U; Priority Program 1656 Intestinal MicrobiotaConsortium/quality assessment participants, GessnerA: Multicenter quality assessment of $16 \mathrm{~S}$ ribosomal DNA-sequencing for microbiome analyses reveals high inter-center variability. Int J Med Microbiol 306: 334-342, 2016.

33. Balvočiūtė M and Huson DH: SILVA, RDP, greengenes, NCBI and OTT-how do these taxonomies compare? BMC Genomics 18 (Suppl 2): S114, 2017.

34. Raetz CR, Ulevitch RJ, Wright SD, Sibley CH, Ding A and Nathan CF: Gram-negative endotoxin: An extraordinary lipid with profound effects on eukaryotic signal transduction. FASEB J 5: 2652-2660, 1991

35. Raetz CR, Guan Z, Ingram BO, Six DA, Song F, Wang X and Zhao J: Discovery of new biosynthetic pathways: The lipid a story. J Lipid Res 50 (Suppl): S103-S108, 2009.

36. Thomas F, Hehemann JH, Rebuffet E, Czjzek M and Michel G: Environmental and gut bacteroidetes: The food connection. Front Microbiol 2: 93, 2011.

37. d'Hennezel E, Abubucker S, Murphy LO and Cullen TW: Total lipopolysaccharide from the human gut microbiome silences Toll-like receptor signaling. mSystems 2: pii: e00046-17, 2017.

38. Santoru ML, Piras C, Murgia A, Palmas V, Camboni T, Liggi S, Ibba I, Lai MA, Orrù S, Blois S, et al: Cross sectional evaluation of the gut-microbiome metabolome axis in an Italian cohort of IBD patients. Sci Rep 7: 9523, 2017.

39. Sun J and Kato I: Gut microbiota, inflammation and colorectal cancer. Genes Dis 3: 130-143, 2016.

40. Guerrero-Preston R, Godoy-Vitorino F, Jedlicka A, Rodríguez-Hilario A, González H, Bondy J, Lawson F, Folawiyo O, Michailidi C, Dziedzic A, et al: 16S rRNA amplicon sequencing identifies microbiota associated with oral cancer, human papilloma virus infection and surgical treatment. Oncotarget 7: 51320-51334, 2016.

41. Jiang S, Xie S, Lv D, Wang P, He H, Zhang T, Zhou Y, Lin Q, Zhou H, Jiang J, et al: Alteration of the gut microbiota in Chinese population with chronic kidney disease. Sci Rep 7: 2870, 2017.

42. Lindberg AA, Weintraub A, Zähringer U and Rietschel ET: Structure-activity relationships in lipopolysaccharides of Bacteroides fragilis. Rev Infect Dis 12 (Suppl 2): S133-S241, 1990.

43. Ramachandran G: Gram-positive and gram-negative bacterial toxins in sepsis: A brief review. Virulence 5: 213-218, 2014

44. Lukiw WJ: Bacteroides fragilis lipopolysaccharide and inflammatory signaling in alzheimer's disease. Front Microbiol 7: 1544, 2016.

45. Couturier MR, Slechta ES, Goulston C, Fisher MA and Hanson KE: Leptotrichia bacteremia in patients receiving high-dose chemotherapy. J Clin Microbiol 50: 1228-1232, 2012.

46. Cekanaviciute E, Yoo BB, Runia TF, Debelius JW, Singh S, Nelson CA, Kanner R, Bencosme Y, Lee YK, Hauser SL, et al: Gut bacteria from multiple sclerosis patients modulate human $\mathrm{T}$ cells and exacerbate symptoms in mouse models. Proc Natl Acad Sci USA 114: 10713-10718, 2017.
47. Hsieh YY, Tung SY, Pan HY, Yen CW, Xu HW, Lin YJ, Deng YF, Hsu WT, Wu CS and Li C: Increased abundance of Clostridium and Fusobacterium in gastric microbiota of patients with gastric cancer in Taiwan. Sci Rep 8: 158, 2018.

48. Apte RN, Pluznik DH and Galanos C: Lipid A, the active part of bacterial endotoxins in inducing serum colony stimulating activity and proliferation of splenic granulocyte/macrophage progenitor cells. J Cell Physiol 87: 71-78, 1976.

49. Lu YC, Yeh WC and Ohashi PS: LPS/TLR4 signal transduction pathway. Cytokine 42: 145-151, 2008.

50. Park BS, Song DH, Kim HM, Choi BS, Lee H and Lee JO: The structural basis of lipopolysaccharide recognition by the TLR4-MD-2 complex. Nature 458: 1191-1195, 2009.

51. McGettrick AF and O'Neill LA: Regulators of TLR4 signaling by endotoxins. Subcell Biochem 53: 153-171, 2010.

52. Oberholzer A, Oberholzer C and Moldawer LL: Sepsis syndromes: Understanding the role of innate and acquired immunity. Shock 16: 83-96, 2001.

53. Grasset E, Puel A, Charpentier J, Collet X, Christensen JE, Tercé $\mathrm{F}$ and Burcelin R: A specific gut microbiota dysbiosis of type 2 diabetic mice induces GLP-1 resistance through an enteric NO-dependent and gut-brain axis mechanism. Cell Metab 25: 1075-1090.e5, 2017.

54. Farhadi A, Banan A, Fields J and Keshavarzian A: Intestinal barrier: An interface between health and disease. J Gastroenterol Hepatol 18: 479-497, 2003.

55. Camilleri M, Madsen K, Spiller R, Greenwood-Van Meerveld B and Verne GN: Intestinal barrier function in health and gastrointestinal disease. Neurogastroenterol Motil 24: 503-512, 2012.

56. Tripathi A, Lammers KM, Goldblum S, Shea-Donohue T, Netzel-Arnett S, Buzza MS, Antalis TM, Vogel SN, Zhao A, Yang S, et al: Identification of human zonulin, a physiological modulator of tight junctions, as prehaptoglobin-2. Proc Natl Acad Sci USA 106: 16799-16804, 2009.

57. Li C, Gao M, Zhang W, Chen C, Zhou F, Hu Z and Zeng C: Zonulin regulates intestinal permeability and facilitates enteric bacteria permeation in coronary artery disease. Sci Rep 6: 29142, 2016.

58. Kelly JR, Kennedy PJ, Cryan JF, Dinan TG, Clarke G and Hyland NP: Breaking down the barriers: The gut microbiome, intestinal permeability and stress-related psychiatric disorders. Front Cell Neurosci 9: 392, 2015.

59. Bischoff SC, Barbara G, Buurman W, Ockhuizen T, Schulzke JD, Serino M, Tilg H, Watson A and Wells JM: Intestinal permeability-a new target for disease prevention and therapy. BMC Gastroenterol 14: 189, 2014

60. Wang L, Llorente C, Hartmann P, Yang AM, Chen P and Schnabl B: Methods to determine intestinal permeability and bacterial translocation during liver disease. J Immunol Methods 421: 44-53, 2015.

61. Fukui H, Brauner B, Bode JC and Bode C: Plasma endotoxin concentrations in patients with alcoholic and non-alcoholic liver disease: Reevaluation with an improved chromogenic assay. J Hepatol 12: 162-169, 1991

62. de Rekeneire N, Peila R, Ding J, Colbert LH, Visser M, Shorr RI, Kritchevsky SB, Kuller LH, Strotmeyer ES, Schwartz AV, et al: Diabetes, hyperglycemia, and inflammation in older individuals: The health, aging and body composition study. Diabetes Care 29:1902-1908, 2006

63. Anderson AJ and Vingrys AJ: Small samples: Does size matter? Invest Ophthalmol Vis Sci 42: 1411-1413, 2001.

This work is licensed under a Creative Commons Attribution-NonCommercial-NoDerivatives 4.0 International (CC BY-NC-ND 4.0) License. 